\title{
Curcumin analog WZ35 induced cell death via ROS-dependent ER stress and G2/M cell cycle arrest in human prostate cancer cells
}

\author{
Xiuhua Zhang 1,2,3†, Minxiao Chen ${ }^{2,3 \dagger}$, Peng Zou ${ }^{2}$, Karvannan Kanchana ${ }^{2}$, Qiaoyou Weng ${ }^{2,4}$, Wenbo Chen ${ }^{2}$, \\ Peng Zhong ${ }^{2}$, Jiansong $\mathrm{Ji}^{4}$, Huiping Zhou ${ }^{2}$, Langchong He ${ }^{1 *}$ and Guang Liang ${ }^{2^{*}}$
}

\begin{abstract}
Background: Prostate cancer is the most commonly diagnosed malignancy among men. The Discovery of new agents for the treatment of prostate cancer is urgently needed. Compound WZ35, a novel analog of the natural product curcumin, exhibited good anti-prostate cancer activity, with an $I_{50}$ of $2.2 \mu \mathrm{M}$ in PC-3 cells. However, the underlying mechanism of WZ35 against prostate cancer cells is still unclear.

Methods: Human prostate cancer PC-3 cells and DU145 cells were treated with WZ35 for further proliferation, apoptosis, cell cycle, and mechanism analyses. NAC and CHOP siRNA were used to validate the role of ROS and ER stress, respectively, in the anti-cancer actions of WZ35.

Results: Our results show that WZ35 exhibited much higher cell growth inhibition than curcumin by inducing ER stress-dependent cell apoptosis in human prostate cells. The reduction of CHOP expression by siRNA partially abrogated WZ35-induced cell apoptosis. WZ35 also dose-dependently induced cell cycle arrest in the G2/M phase. Furthermore, we found that WZ35 treatment for 30 min significantly induced reactive oxygen species (ROS) production in PC-3 cells. Co-treatment with the ROS scavenger NAC completely abrogated the induction of WZ35 on cell apoptosis, ER stress activation, and cell cycle arrest, indicating an upstream role of ROS generation in mediating the anti-cancer effect of WZ35.

Conclusions: Taken together, this work presents the novel anticancer candidate WZ35 for the treatment of prostate cancer, and importantly, reveals that increased ROS generation might be an effective strategy in human prostate cancer treatment.
\end{abstract}

Keywords: Cell cycle arrest, CHOP, Curcumin analog, ER stress, Prostate cancer, PC-3, ROS

\section{Background}

Prostate cancer is the most commonly diagnosed malignancy among men in industrialized countries, accounting for the second leading cause of cancer-related death. Conventional therapies produce a high rate of cure for patients with localized prostate cancer by surgical therapy, but there is no cure once the disease has spread beyond the prostate. Traditionally, the treatment of

\footnotetext{
*Correspondence: helc@mail.xjtu.edu.cn; wzmcliangguang@163.com ${ }^{\dagger}$ Equal contributors

'School of Pharmacy, Health Science Center, Xi'an Jiaotong University, Xi'an, 710061 Shanxi, China

${ }^{2}$ Chemical Biology Research Center, School of Pharmaceutical Sciences, Wenzhou Medical Universtiy, Wenzhou 325035, Zhejiang, China Full list of author information is available at the end of the article
}

prostate cancer has been based on the deprivation of androgens to the developing tumor. Though initially successful, this form of therapy fails in advanced stages of the disease, as the cells develop the ability to sustain growth and proliferation even in the absence of androgens, thus acquiring androgen resistance [1]. In addition, these tumors tend to be highly resistant to conventional cytotoxic agents such as cisplatin. Presently available treatments for advanced hormone-resistant prostate cancer are marginally effective; thus, new agents are needed to selectively kill cancer cells.

Curcumin, a polyphenolic compound that is extracted from the rhizome of the plant Curcuma longa, has become a focus of interest regarding its antitumor effects 
in multiple cancer cell types including prostate cancer cells [2]. Moreover, curcumin is under clinical trials mainly for cancer related diseases [3, 4]. Interestingly, phase1 clinical trials already demonstrated the safety of curcumin even at high doses (12 g/day). However, the clinical advancement of this promising natural compound is hampered by its poor water solubility and short biological half-life, resulting in low bioavailability in both plasma and tissues [5]. Multiple approaches are being sought to overcome these limitations. In the past several years, our lab has focused on the chemical modification of curcumin to find novel molecules for drug development $[6,7]$. Previously, a series of mono-carbonyl analogs of curcumin were synthesized and evaluated against prostate cancer cells. Among them, compound WZ35 (Fig. 1a) exhibited good anti-prostate cancer activity at the cellular level, with an $\mathrm{IC}_{50}$ of $2.2 \mu \mathrm{M}$, compared to that of curcumin at $20.9 \mu \mathrm{M}$ in PC-3 cells (Fig. 1b), an androgen-resistant and high metastatic potential human prostate cancer cell lines.

Oxidative stress plays an important role in controlling cancer cell behavior. Cancer cells may potentially benefit from oxidative stress induction and the production of reactive oxygen species (ROS), which are known to increase the rate of mutations $[8,9]$. However, the oxidative stress response is a balance between pro-survival and proapoptotic signaling pathways [10]. An uncontrolled highlevel ROS also triggers a series of pro-apoptotic signaling pathways, including endoplasmic reticulum (ER) stress and mitochondrial dysfunction, and ultimately leads to cellular apoptosis [10]. Because cancer cells have a higher level of oxidative stress than non-malignant cells,

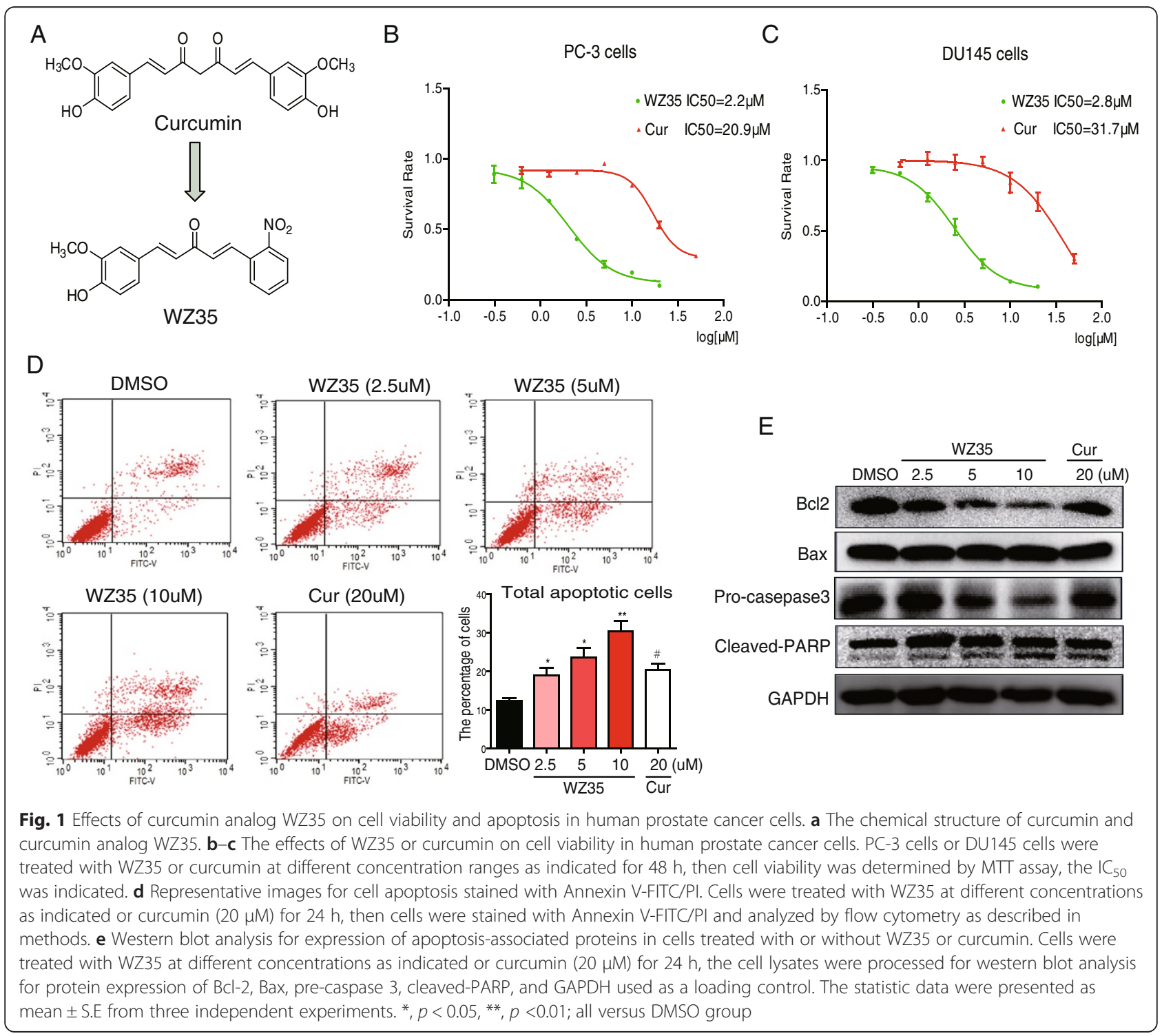


they are vulnerable to the acute induction of oxidative stress that is caused by agents inducing $\operatorname{ROS}[9,11]$. Mounting evidence suggests that increasing oxidative stress might be an effective strategy to eliminate cancer cells. Increased ROS generation and oxidative stress have been reported in prostate cancer cells [11]. Thus, agents that can induce ROS generation may be effective in killing prostate cancer cells.

The aim of this study was to determine the effect and mechanism of WZ35 against prostate cancer cells. Our data demonstrate that WZ35 showed strong antitumor potential against PC-3 cells by activating ROS production and subsequently inducing ER stress-dependent apoptosis and cell cycle arrest.

\section{Methods}

\section{Reagents}

WZ35 ( $>98 \%$ purity) was prepared in our lab using a previously described method. Curcumin, $\mathrm{N}$-acetylcysteine (NAC), glutamine (L-GSH), dimethylsulfoxide (DMSO) and methyl thiazolyl tetrazolium (MTT) were obtained from Sigma-Aldrich (St. Louis, MO). The primary antibodies, including anti-Bcl2 (sc-492), anti-Bax(sc-493), anti-caspase 3 (sc-32577), anti-Cdc2 (sc-54), anti-Cyclin B1 (sc-245), anti-MDM2 (sc-965), anti-GAPDH (sc-32233), anti-p-PERK (sc-32577), horseradish peroxidase (HRP)conjugated (sc-2313) and phycoerythrin (PE)-conjugated (sc-3755) secondary antibodies were purchased from Santa Cruz Biotechnology (Santa Cruz, CA). The primary antibodies, including anti-cleaved PARP (5625S), anti-peIF2 $\alpha$ (3398S), anti-ATF4 (11815S), and anti-CHOP (2895S), were purchased from Cell Signaling Technology (Danvers, MA). CHOP siRNA was purchased from GenePharma (Shanghai, China). FITC Annexin V apoptosis Detection Kit I and propidium iodide (PI) were obtained from BD Pharmingen (Franklin Lakes, NJ). Bradford protein assay kit, polyvinyldene fluoride membrane, ECL kit were obtained from Bio-Rad (Hercules, CA). Lipofectamine 2000, TRIZOL reagent, M-MLV Reverse Transcriptase Kit, PCR Supermix kit and primers for genes, including $\mathrm{CHOP}$ and $\beta$-actin, were purchased from Invitrogen Life Technology (Carlsbad, CA). DCFH-DA was obtained from Beyotime Biotech (Nantong, China).

\section{Cell culture}

Human prostate cancer PC-3 cells and DU145 cells were obtained from the Shanghai Institute of Life Sciences Cell Resource Center (Shanghai, China) and cultured in DMEM/F12 medium (Gibco, Eggenstein, Germany) that was supplemented with $10 \%$ heat-inactivated FBS (Hyclone, Logan, UT), $100 \mathrm{U} / \mathrm{mL}$ penicillin and $100 \mu \mathrm{g} / \mathrm{mL}$ streptomycin (Mediatech Inc., Manassas, VA) in a humidified atmosphere of $5 \% \mathrm{CO}_{2}$ at $37^{\circ} \mathrm{C}$.

\section{Methyl Thiazolyl Tetrazolium (MTT) assay}

All of the experiments were carried out $24 \mathrm{~h}$ after the cells were seeded. The tested compounds were dissolved in DMSO and diluted with DMEM/F12 medium at different concentrations. The tumor cells were incubated with test compounds for $48 \mathrm{~h}$ before the MTT assay. A fresh solution of MTT $(5 \mathrm{mg} / \mathrm{mL})$ that was prepared in PBS was added to each single well of the 96-well plate. The plate was then incubated in a $\mathrm{CO}_{2}$ incubator for $4 \mathrm{~h}$. Formazan cyrstals that formed in living cells was dissolved in $150 \mu \mathrm{L}$ of dimethyl sulfoxide, and the absorbance of the solution was measured at $490 \mathrm{~nm}$ using a microplate reader (Reader 400 SFC, LabInstruments, Hamburg,Germany). The $\mathrm{IC}_{50}$ values were calculated using the GraphPad Prism 5 software.

\section{Measurement of cell apoptosis}

Apoptosis was analyzed by Annexin V-FITC/PI staining. Briefly, after treatment, the cells were harvested and washed with PBS followed by the addition of $1 \times$ binding buffer $(500 \mu \mathrm{l})$ and Annexin V-FITC $(2 \mu \mathrm{l})$, incubated at RT in the dark for $20 \mathrm{~min}$ and centrifuged. The cell pellet was re-suspended in $1 \times$ binding buffer, added with $3 \mu \mathrm{l}$ of PI $(30 \mu \mathrm{g} / \mathrm{ml})$ and acquired immediately on an FACS Caliber flow cytometer (BD Biosciences, CA). An analysis was performed for Annexin V-FITC binding using the FITC signal detector (FL-1) and PI staining by the phycoerythrin emission signal detector (FL-2) using the CellQuest ${ }^{\mathrm{m}}$ software (BD Biosciences, CA) or the FlowJo 7.6 software (TreeStar, San Carlos, CA).

\section{Western blot assay}

After treatment, the cells were collected and extracted for total proteins. The protein concentrations in all of the samples were determined using the Bradford protein assay kit. Protein samples $(30-100 \mu \mathrm{g})$ were subjected to (10-15\%) sodium dodecyl sulfate-polyacrylamide gel electrophoresis, and transferred onto polyvinyldene fluoride membrane. After being blocked in blocking buffer (5\% milk in tris-buffered saline containing $0.05 \%$ Tween 20) for $1.5 \mathrm{~h}$ at room temperature, membranes were incubated with different primary antibodies overnight at $4{ }^{\circ} \mathrm{C}$. Then, the membranes were washed in TBST and reacted with secondary horseradish peroxidase-conjugated antibody for $1 \mathrm{~h}$ at room temperature, and the immunoreactive bands were visualized using an ECL kit. The density of the immunoreactive bands was analyzed using Image J computer software (National Institute of Health, MD).

\section{Determination of Reactive Oxygen Species (ROS) production}

Intracellular ROS generation was monitored by an FACS Caliber flow cytometer (BD Biosciences, CA) using the peroxide-sensitive fluorescent probe $2^{\prime}, 7^{\prime}$-dichlorofluorescin 
diacetate (DCFH-DA) as previously described [12]. In brief, after treatment, the cells were incubated with $10 \mu \mathrm{M}$ DCFH-DA at $37{ }^{\circ} \mathrm{C}$ for $30 \mathrm{~min}$, resuspended in ice-cold phosphate buffered saline (PBS) and placed on ice in a dark environment. The intracellular peroxide levels were measured by an FACS Caliber flow cytometer that emitted a fluorescence signal at $525 \mathrm{~nm}$. Each group was acquired for 10,000 individual cells using the CellQuest ${ }^{\mathrm{Tm}}$ software (BD Biosciences, CA) and analyzed by the FlowJo 7.6 software (TreeStar, San Carlos, CA).

\section{RT-qPCR assay}

The total mRNA was isolated from cells using TRIZOL Reagent according to the manufacturer's instructions. Reverse transcription and quantitative PCR were performed using the M-MLV Reverse Transcriptase Kit and PCR Supermix kit according to the manufacturer's instructions. Real-time qPCR was carried out using the Eppendorf Real plex 4 instrument (Eppendorf, Hamburg, Germany). The relative amount of each gene was normalized to the amount of $\beta$-actin. The primer sequences used were shown as followed. Human CHOP: forward, CAGAACCAGCAG AGGTCACA; reverse, GCTGTG CCACTTTCCTTTC. Human $\beta$-action: forward, TCCT TCCTGGGCATGGAGTC; reverse, GTAACGCAACT AAGTCATAGTC.

\section{Transient transfection of small interfering RNA (siRNA)}

The cells were transfected with siRNA $(50 \mathrm{pmol} / \mathrm{ml}) \mathrm{tar}-$ geting CHOP or non-targeted siRNA as a control using the lipofectamine 2000 reagent as described by the manufacturer's instructions. Subsequently, the transfected cells were washed and changed with complete media and used in further studies. The siRNA sequences used were as followed: Human control siRNA (CtrlsiRNA): sense, 5' -AGUACUGCUUACGAUACGGTT-3'; antisense, 5'-CCGUAUCGUAAGCAGUACUTT-3'. CHOP siRNA: sense, 5'-CCAGGAAACGGACAACAGAGTT-3'; antisense, 5'-CUCUGUUUCCGUUUCCUGGTT-3'. These siRNA sequences were adopted according to previous published work [13].

\section{Cell cycle analysis}

The cell cycle status and nuclear DNA contents were determined using propidium iodide (PI) staining and flow cytometry. Briefly, the cells were collected, fixed with $75 \%$ ice-cold ethanol and stored at $4{ }^{\circ} \mathrm{C}$ for $24 \mathrm{~h}$. After they were washed with PBS, the cells were stained with PI [50 $\mu \mathrm{g} / \mathrm{ml}$ PI and $10 \mu \mathrm{g} / \mathrm{ml}$ ribonuclease (RNase) in PBS] at $4{ }^{\circ} \mathrm{C}$ for $20 \mathrm{~min}$ in the dark. The cells were washed and subjected to an FACS Caliber flow cytometric analysis of the DNA content. The cell fractions in the G2/M phase were used for statistical analysis using the FlowJo 7.6 software (TreeStar, San Carlos, CA).

\section{Immunofluorescence assay for CHOP}

Cells were fixed with $4 \%$ paraformaldehyde and permeabilized with $100 \%$ methanol at $-20{ }^{\circ} \mathrm{C}$ for $5 \mathrm{~min}$. After fixation and permeabilization, the cells were washed twice with PBS containing $1 \% \mathrm{BSA}$ and then incubated with the primary antibody for $\mathrm{CHOP}$ overnight at $4{ }^{\circ} \mathrm{C}$, followed by incubation with the PE-conjugated secondary antibody. Then, the cells were counterstained with DAPI and viewed under a Nikon fluorescence microscope (400x amplification; Nikon, Japan).

\section{Statistical analysis}

All of the experiments were performed independently three times. The data are presented as means \pm SE. The statistical significance of differences between groups was obtained by the student's $t$-test or ANOVA multiple comparisons in GraphPad Pro (GraphPad, San Diego, $C A$ ). Differences were considered significant at *, $P<0.05$; **, $P<0.01$; ***, $P<0.001$.

\section{Results}

WZ35 reduced cell viability and induced cell apoptosis in human prostate cancer cells

To determine the cytotoxic effects of WZ35 in prostate cancer cell lines, an MTT assay was performed to evaluate the viability in human prostate cancer PC-3 and DU145 cells. As shown in Fig. 1b and c, WZ35 or curcumin treatment significantly decreased the viability of PC-3 cells and DU145 cells in a dose-dependent manner. The $\mathrm{IC}_{50}$ value for WZ35 and curcumin was $2.2 \mu \mathrm{M}$ versus $20.9 \mu \mathrm{M}$ in PC-3 cells, and 2.8 versus $31.7 \mu \mathrm{M}$ in DU145 cells, respectively, indicating a much better anticancer ability of WZ35 than curcumin. We then evaluated the role of apoptosis in WZ35-induced cell death using Annexin V-FITC/PI staining. A time-course assay revealed that the occurrence of cell apoptosis induced by WZ35 began at $12 \mathrm{~h}$ and peaked at $24 \mathrm{~h}$ after WZ35 treatment (Additional file 1: Figure S1A). The exposure of PC-3 cells to WZ35 at various concentrations for $24 \mathrm{~h}$ dosedependently increased the number of apoptotic cells (Fig. 1d). In addition, WZ35 was dramatically more effective than curcumin in apoptosis induction. The levels of apoptosis-associated proteins were also examined by western blot analysis in PC-3 cells. As shown in Fig. 1e, WZ35 treatment decreased the protein level of $\mathrm{Bcl}-2$ and procaspase 3 and increased the cleaved PARP in a dosedependent manner but had no effect on Bax expression. No obvious changes were observed in these protein levels in cells that were treated with $20 \mu \mathrm{M}$ curcumin (Fig. 1e).

\section{ROS overproduction mediated WZ35-induced apoptosis in PC-3 cells}

We investigated whether intracellular ROS generation was implicated in the anti-cancer effects of WZ35. The 
ROS level was assessed by using fluorescent probe DCFH-DA that detected $\mathrm{H}_{2} \mathrm{O}_{2}$. Interestingly, WZ35 treatment significantly increased intracellular ROS generation in a time-dependent manner (Fig. 2a). Furthermore, co-treatment with $\mathrm{N}$-acetylcysteine (NAC), an ROS scavenger, significantly inhibited WZ35-induced ROS generation (Fig. 2b). These data show that WZ35 could induce the accumulation of ROS in prostate cancer cells. We then examined whether increased ROS was required for cell apoptosis induced by WZ35. As shown in Fig. 2c, co-treatment with NAC at the concentration of $10 \mathrm{mM}$ almost completely abrogated WZ35-induced cell apoptosis. In addition, reversed cell apoptosis was also observed in WZ35-treated cells in the presence of glutathione (L-GSH), another potent antioxidant that has been widely used to define the function of ROS in numerous biological and pathological processes (Fig. 2d). Collectively, these results indicated that ROS generation plays a central role in mediating WZ35-induced cell apoptosis.

\section{WZ35-induced cell apoptosis through ER stress-mediated CHOP expression in PC-3 cells}

ROS generation has been reported to activate multiple pro-apoptotic cascades, including the ER stress-induced cancer cell apoptosis pathway $[10,14]$. Thus, we

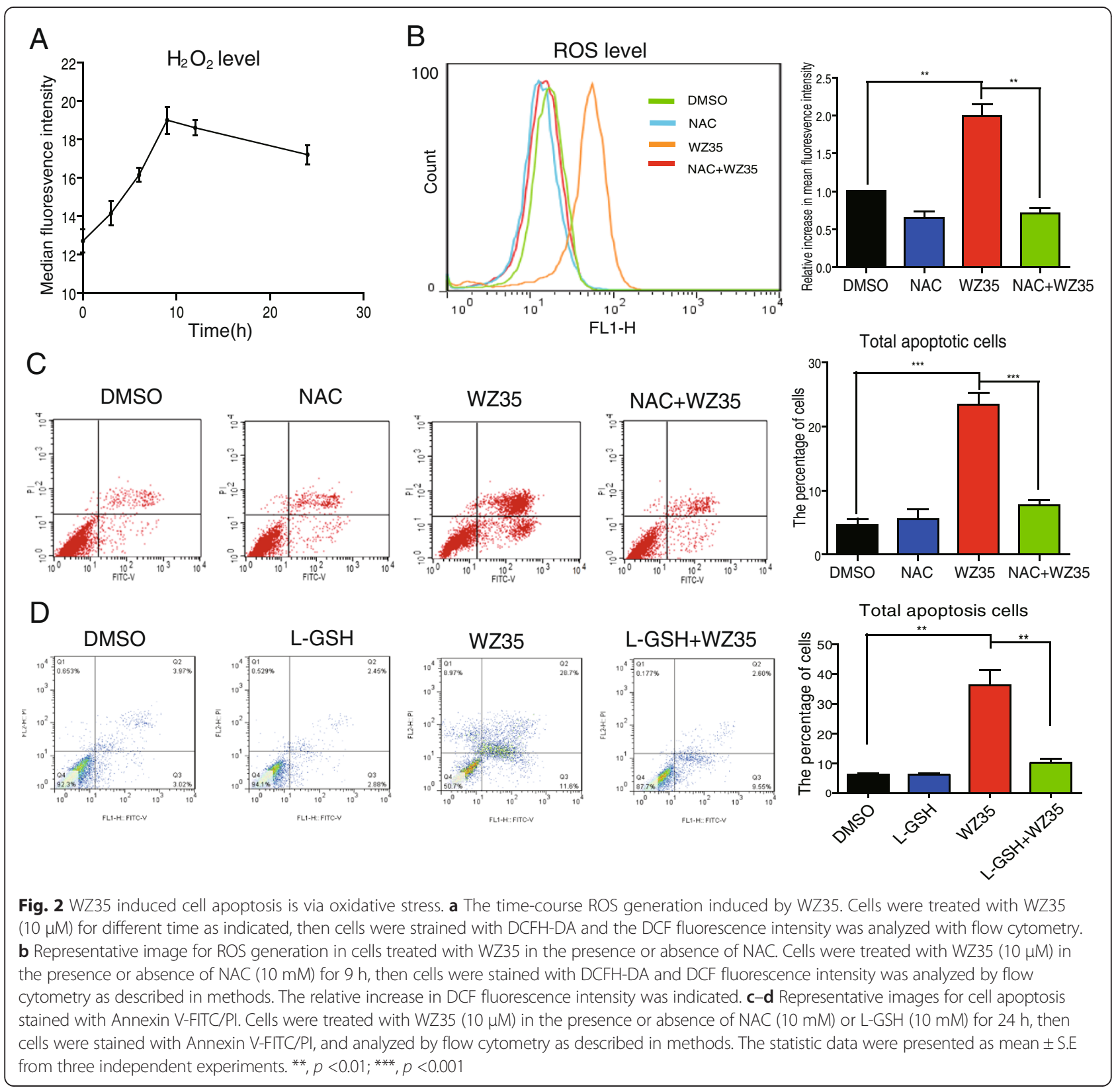


determined the effects of treatment with WZ35 on the induction of ER stress. When PC-3 cells were treated with WZ35 for various time intervals, we noticed a transient increase in the level of phosphorylated PERK, commencing after $2 \mathrm{~h}$ of treatment with WZ35 and remaining elevated for up to $4 \mathrm{~h}$ (Fig. 3a). WZ35 treatment also induced a constant increase in the level of phosphorylated eIF2 $\alpha 3$ to $12 \mathrm{~h}$ after WZ35 treatment (Fig. 3a). ATF4 expression also increased in a similar manner with p-eIF2 $\alpha$ (Fig. 3a).

CHOP is considered a marker of the commitment of ER stress-induced apoptosis. Western blotting analysis further showed that $\mathrm{CHOP}$ protein expression apparently increased 9-24 h after WZ35 treatment and peaked at $12 \mathrm{~h}$ (Fig. 3a). Similar time-course results were also observed in the mRNA level of CHOP induction (Fig. 3b). Figure 3c shows that compound WZ35 induced CHOP mRNA up-regulation in a dose-dependent manner. These results suggest that WZ35 can induce ER stress in prostate cancer cells. In order to further confirm that ER stress plays an important role in the induction of PC-3 cell apoptosis by WZ35, we constructed the siRNA for CHOP gene silencing. PC-3 cells were transfected with the CHOP siRNA sequence or the control

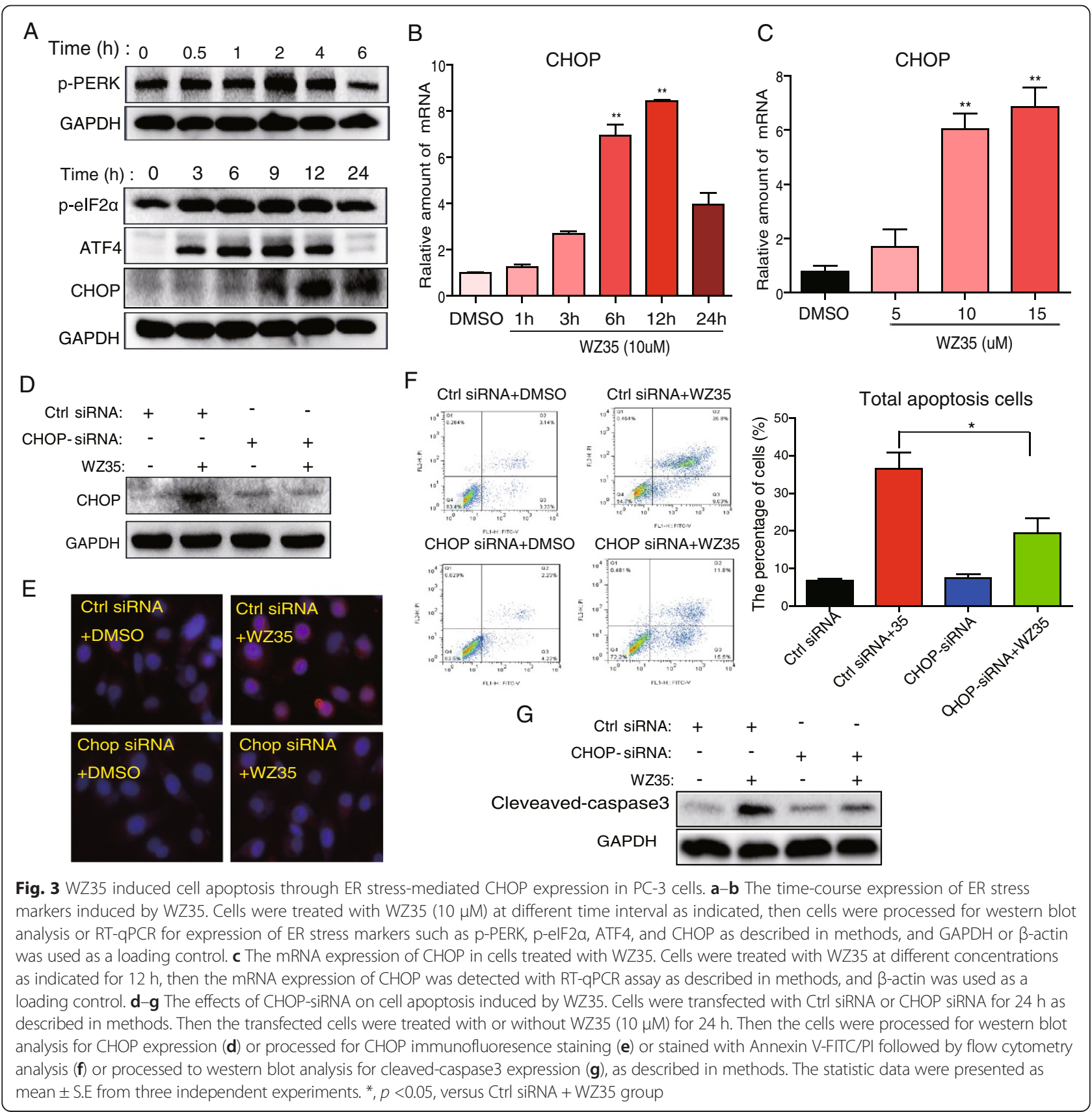


sequence. Western blot analysis demonstrated that the transfection of CHOP siRNA resulted in a significant decrease in CHOP expression in WZ35-treated PC-3 cells (Fig. 3d), compared to cells that were transfected with control scrambled siRNA. This result was further confirmed by immunofluorescence staining (Fig. 3e). Furthermore, to confirm that a reduction of $\mathrm{CHOP}$ expression inhibits WZ35-induced PC-3 cell apoptosis, we treated CHOP siRNA-transfected PC-3 cells with WZ35. Figure $3 \mathrm{f}$ shows that when CHOP expression in PC-3 cells was silenced, cell apoptosis induced by WZ35 was significantly reduced compared to that of the control group $(P<0.05)$. Furthermore, the protein level of cleaved-caspase 3 induced by WZ35 was also reduced in CHOP-knockdown PC3 cells (Fig. 3g). Taken together, these results indicate that WZ35-induced cell apoptosis is, at least partly, mediated by the ER stress pathway.

\section{WZ35 induced cell cycle arrest in G2/M phase in PC-3 cells}

To determine the anti-mitogenic effect of WZ35, we performed a cell-cycle analysis in PC-3 cells. As shown in Fig. 4a, WZ35 treatment induced the accumulation of cells in the G2/M phase in a dose-dependent manner. The effect of $10 \mu \mathrm{M}$ WZ35 on cell cycle arrest was stronger than that of curcumin at $20 \mu \mathrm{M}$ (Fig. 4a). In addition, the cell population in S phase were reduced, in association with the increased cell population in the G2/ $M$ phase, and no changes were observed in the G0 phase (Additional file 1: Figure S1B). We further tested the effects of WZ35 on cell cycle arrest-related proteins by western blot analysis. G2/M transition is regulated by the cyclin B1/CDC2 complex [15], and MDM2 is a negative regulator of p21, which is involved in the $\mathrm{G} 2 / \mathrm{M}$ checkpoint and is required for cell cycle arrest in the G2/M2 phase [16, 17]. Figure $4 \mathrm{~b}-\mathrm{d}$ reveals that WZ35 treatment decreased the protein level of $\mathrm{CDC} 2$, Cyclin B1, and MDM2 in a dose-dependent manner, while curcumin treatment at $20 \mu \mathrm{M}$ has no significant effects on these proteins. These results suggest an anti-mitogenic effect of WZ35 in PC-3 cells.

\section{Both ER stress and G2/M arrest induced by WZ35 were mediated by ROS generation in PC-3 cells}

Because ROS production induced by WZ35 occurs within a relatively much earlier time (30 min), compared to ER stress activation and cell cycle arrest, we supposed that ROS generation might be the upstream incidence in the procedure of WZ35-induced PC-3 cell death. Thus, we determined whether ROS generation is required for WZ35-induced ER stress and G2/M arrest in PC-3 cells. As shown in Fig. 5a, co-treatment with the ROS

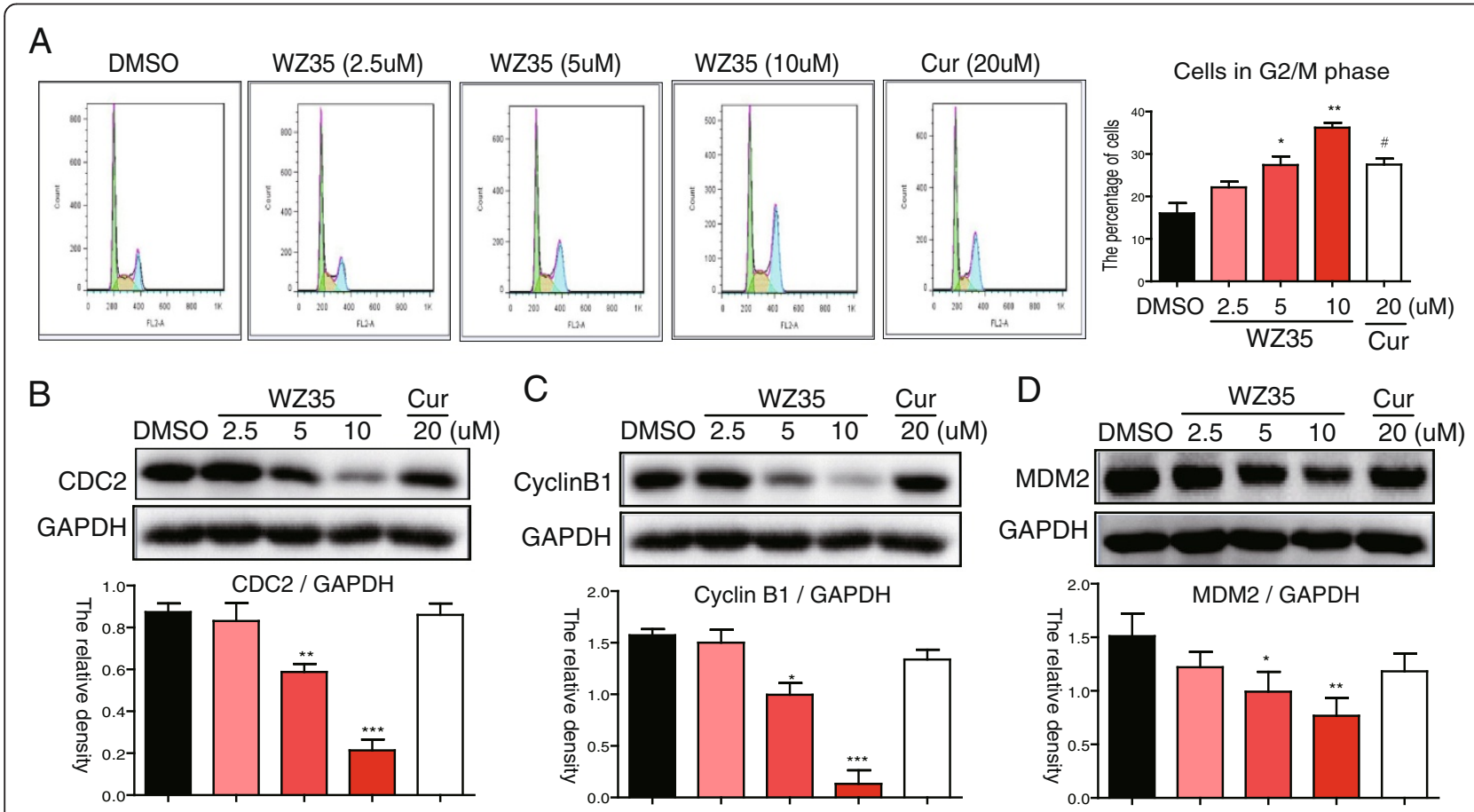

Fig. 4 WZ35 induced cell cycle arrest in the G2/M phase in PC-3 cells. Cells were treated with WZ35 at different concentrations as indicated or curcumin $(20 \mu \mathrm{M})$ for $24 \mathrm{~h}$, then cells were processed for cell cycle analysis (a) or western blot analysis for expression of cell cycle-associated proteins (b-d) as described in methods. The statistic data were presented as mean \pm S.E from three independent experiments. ${ }^{*}, p<0.05,{ }^{* *}$, $p<0.01$; ${ }^{* *}, p<0.001$; all versus DMSO group 


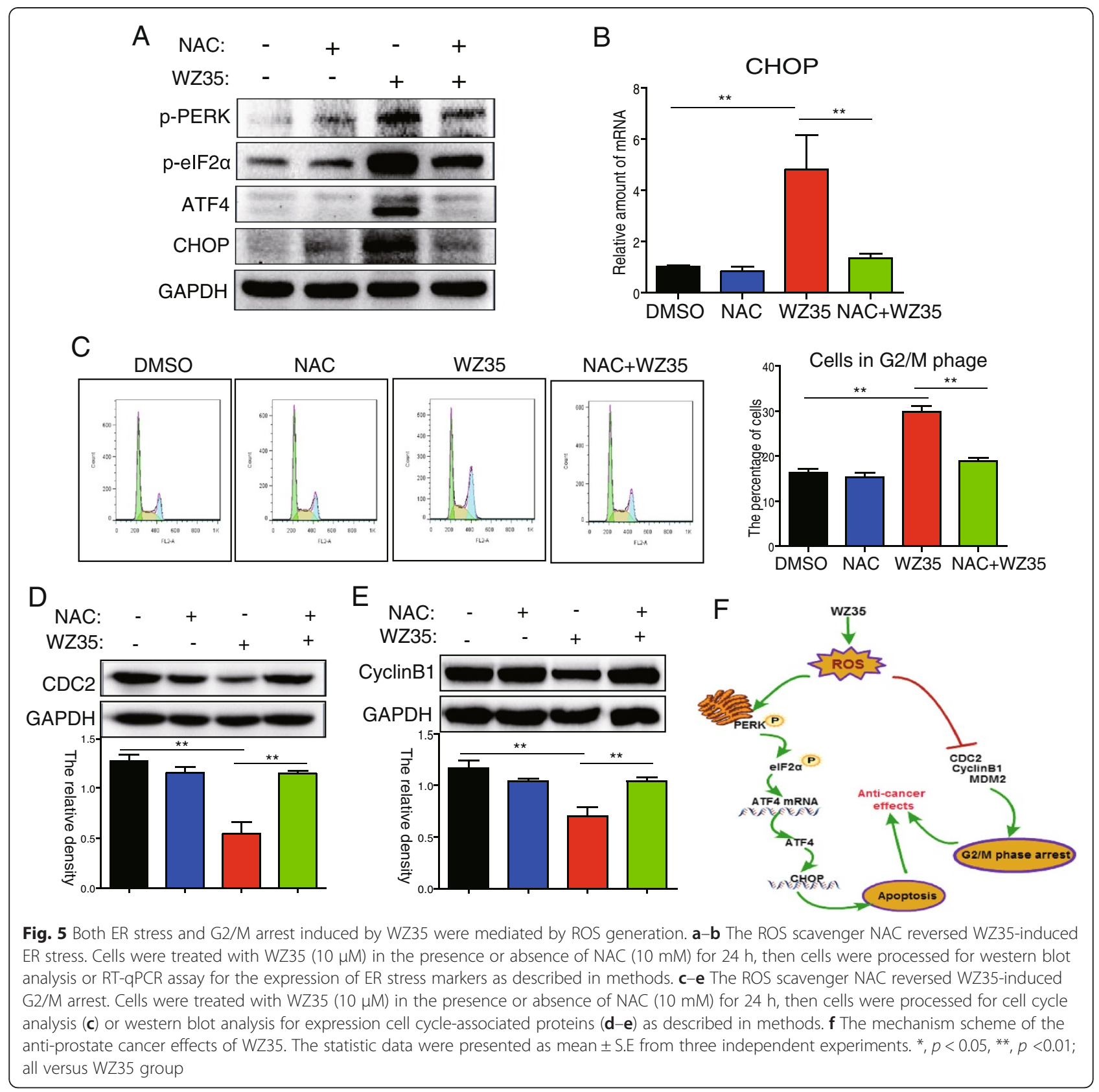

scavenger NAC markedly inhibited the WZ35-induced over-expression of ER stress markers. A similar result was also observed at the mRNA level of CHOP induced by WZ35 in the presence of NAC (Fig. 5b). These results indicate that WZ35-induced ER stress is mediated by increased oxidative stress. As expected, co-treatment with NAC also significantly inhibited the WZ35-induced accumulation of cells in the G2/M phase (Fig. 5c). In agreement, western blot analysis revealed that cotreatment with NAC significantly reversed the decreased protein level of CDC2 and CyclinB1 induced by WZ35 in PC-3 cells (Fig. 5d and e).

\section{Discussion}

In the present study, we demonstrated that a new novel curcumin analog WZ35 showed excellent anticancer effects in prostate cancer cells via inducing ROSdependent ER stress and G2/M cell cycle arrest. These findings indicate that WZ35 should be further explored as an effective anticancer agent for the treatment of prostate cancer. Accumulating evidence suggests that increasing oxidative stress might be an effective strategy to eliminate cancer cells [18]. Agents with the potential to induce ROS generation have anticancer effects in prostate cancer cells, such as salinomycin [19], Diallyl trisulfide 
[20] and WZC02-9 [21]. Herein, increased ROS generation was also observed in WZ35-treated PC-3 cells (Fig. 2). Importantly, the abrogation of ROS production by NAC co-treatment almost completely reversed the WZ35induced cell apoptosis, suggesting the significant involvement of ROS in WZ35-induced cell death. Thus, our results further indicate that developing agents with inducing ROS potential will be a good strategy for cancer therapy.

Recently, ER stress-induced cancer cell apoptosis has become a novel signaling target for the development of cancer therapeutic drugs [22-24]. Various pathological conditions, such as hypoxia, ER-Ca2 ${ }^{+}$depletion, oxidative injury, hypoglycemia and viral infections, may cause an imbalance between the protein folding load and capacity; this cellular condition is known as ER stress [25]. The initial role of ER stress is tailored to re-establish ER homeostasis. However, when ER stress is too severe or cannot be solved, it changes from a pro-survival to a pro-death response, culminating in the activation of intrinsic apoptosis [26]. The most important pathway translating the cell from ER stress to death is the PERK/ eIF2 $\alpha$ pathway [14]. The phosphorylation of PERK/eIF2 $\alpha$ subsequently induces the expression of transcription factors ATF4 and CHOP, which are important elements triggering the pro-apoptotic signaling [27]. Here, we found that WZ35 induced PERK/eIF2 $\alpha$ activation and ATF4/CHOP expression in a time-course manner, indicating that WZ35 activated pro-apoptotic ER stress signaling (Fig. 3a-c). Then, we found that WZ35-mediated apoptosis was partially reduced in CHOP-deficient cells, confirming the mediation of ER stress in WZ35-induced cell apoptosis (Figure F). Similar to that of WZ35, targeting ER stress to induce cancer cell death has been reported by our previous works for other monocarbonyl analogs of curcumin such as B19 [28], B63 [29] and B82 [30].

Increased oxidative stress is linked to ER stress activation [25]. ROS could exacerbate protein misfolding in the ER lumen by oxidizing amino acids in folding proteins or by modifying chaperone and/or ERAD function, thereby amplifying unfolding protein response (UPR) signaling [31]. Increased ROS generation or oxidative stress may be responsible for subsequent ER stress and ER stress-dependent cell apoptosis. Antioxidants reduce ER stress and improve cell survival [32]. However, an inverse relationship between oxidative stress and ER stress has also been reported by Jypti D et al. [32]. The authors found that the accumulation of unfolded protein in the ER lumen is sufficient to produce ROS and suggested that unfolded protein in the ER lumen signalsROS production as a second messenger to activate the UPR and induce apoptosis [32]. In this study, we found that ER stress activated by WZ35 was almost completely reversed by the presence of NAC, a ROS scavenger (Fig. 5).
In addition, WZ35 treatment activated ROS generation within $30 \mathrm{~min}$, much earlier than the treatment time for ER stress induction. Thus, these results indicate that the increased ROS generation induced by WZ35 is required for the induction of ER stress and triggers ER stressdependent apoptosis in PC-3 cells.

The present study also shows that WZ35 treatment inhibited G2/M progression in prostate cancer cells, in association with the decreased expression of $\mathrm{CDC} 2$, cyclinB1, and MDM2 (Fig. 4). As each phase of the cell cycle is driven by specific CDKs, the CDK1 (also named CDC2)-cyclin B1 complex is responsible for driving cells through mitosis [15]. MDM2 is an oncoprotein that can regulate the cell cycle and is a negative regulator of $\mathrm{p} 21$, which is required for cell cycle arrest in the G2/M2 phase [16, 17]. Several studies have reported that some compounds inducing ROS generation, including diallyl trisulfide [23], plumbagin [33], and diallyl disulfide [25], could induce cell cycle arrest at the G2/M phase in several cancer cells. Here, we also found the WZ35-induced G2/M arrest was reversed by co-treatment with an ROS scavenger (Fig. 5). These results suggest a strong link between oxidative stress and cell cycle arrest. Although this link has been also observed in other anti-cancer therapies, the underling mechanism has not been totally resolved. Some studies reported that p21 plays a vital role in mediating ROS-induced G2/M arrest [34]. In addition, a p21-independent mechanism has also been reported [20]. Xiao et al. revealed that increased ROS can induce the destruction and hyperphosphorylation of CDC25c, a phosphatases that can dephosphorylate CDK1 in Thr14 and Tyr15, and hence activate the CDK1/ cyclinB1 kinase complex [20]. Although our study demonstrates that the ROS-induced cell cycle arrest is associated with $\mathrm{CDC} 2 /$ cyclin $\mathrm{B} 1$ reduction, the exact mechanism requires further research.

The excellent anticancer effects of WZ35 suggest the potential advantages of the mono-carbonyl structure of curcumin. Our group has been engaged in designing and discovering new small molecules from natural curcumin. Previously, some other monocarbonyl analogs of curcumin, such as B19 [28], B63 [29], and B82 [30], that were synthesized by our lab were demonstrated to possess strong anti-cancer effects in various cancer cells. In addition, we investigated the mechanism by which these compounds induce cancer cell death. As previously reported, one common mechanism underling their anticancer action is that all of these agents could induce lethal ER stress. This result is also observed in the anti-cancer effects of WZ35. However, our previous studies have failed to demonstrate how curcumin analogs activate ER stress. In the present study, we found that WZ35-increased ROS generation occurred upstream of ER stress. This mechanism may be also 
suitable for previously reported mono-carbonyl analogs of curcumin.

In addition, similar results were observed in DU145 cells. As shown in Additional file 1: Figure S2, we found that WZ35 also induced ROS accumulation, CHOP expression, cell apoptosis and G2/M arrest in DU145 cells. More importantly, all of these alterations were significantly inhibited by ROS scavenger NAC pretreatment, and the genetic silence of $\mathrm{CHOP}$ by siRNA approach also remarkably prevented cell apoptosis in DU145 cells. Collecting the data from DU145 and PC-3 cells, our results vividly demonstrate the pivotal role of the ROS-ER stress signaling pathway in mediating the anticancer effects of WZ35 in prostate cancer cells.

\section{Conclusions}

In summary, a new monocarbonyl analog of curcumin, WZ35, exhibited antitumor effects on human PC-3 and DU145 cells in vitro by inducing ROS generation and subsequent ER stress and G2/M cycle arrest. The discovery of the activation of ROS-mediated apoptosis by the curcumin analog WZ35 may provide new strategy for curcumin-based anticancer drug design and development. The new compound WZ35 could be further explored as a potential anticancer agent for the treatment of prostate cancer.

\section{Additional file}

Additional file 1: The anti-cancer effects of WZ35 treatment in PC3 and DU145 cells. Figures S1, The time-course of cell apoptosis and cell cycle progression in response to WZ35 treatment in PC-3 cells. and S2, The effects of WZ35 treatment in DU145 cells.(DOC $290 \mathrm{~kb})$

\section{Competing interests}

The authors declare that they have no competing interests.

\section{Authors' contributions}

$\mathrm{GL}$ and $\mathrm{LH}$ conceived and designed the experiments. $\mathrm{XZ}, \mathrm{MC}$, and $\mathrm{PZ}$ carried out the experiments. KK, QW, WC, and PZ performed the data collection and analysis. $\mathrm{HZ}, \mathrm{JJ}$, and $\mathrm{PZ}$ reviewed the manuscript. All authors had read and approved the final manuscript.

\section{Acknowledgements}

The work was supported by National Natural Science Foundation of China (81173140, 81270489, 81573657, and 81503107), Zhejiang Province Natural Science Funding of China (LY13H160022), and Zhejiang Key Health Science and Technology Project (WKJ2013-2-021).

\section{Author details \\ 'School of Pharmacy, Health Science Center, Xi'an Jiaotong University, Xi'an, 710061 Shanxi, China. ${ }^{2}$ Chemical Biology Research Center, School of Pharmaceutical Sciences, Wenzhou Medical Universtiy, Wenzhou 325035, Zhejiang, China. ${ }^{3}$ Department of Pharmacy, the First Affiliated Hospital of Wenzhou Medical University, Wenzhou 325035, Zhejiang, China. ${ }^{4}$ Department of Interventional Radiology, The Fifth Affiliated Hospital of Wenzhou Medical University, Lishui 323000, Zhejiang, China.}

Received: 18 May 2015 Accepted: 26 October 2015

Published online: 06 November 2015

\section{References}

1. Zoubeidi A, Zardan A, Beraldi E, Fazli L, Sowery R, Rennie P, et al. Cooperative interactions between androgen receptor (AR) and heat-shock protein 27 facilitate AR transcriptional activity. Cancer Res. 2007;67(21):10455-65.

2. Teiten M-H, Gaascht F, Eifes S, Dicato M, Diederich M. Chemopreventive potential of curcumin in prostate cancer. Genes Nutrit. 2010;5(1):61-74.

3. Johnson JJ, Mukhtar H. Curcumin for chemoprevention of colon cancer. Cancer Lett. 2007;255(2):170-81.

4. Dhillon N, Aggarwal BB, Newman RA, Wolff RA, Kunnumakkara AB, Abbruzzese JL, et al. Phase II trial of curcumin in patients with advanced pancreatic cancer. Clin Cancer Res. 2008;14(14):4491-9.

5. Anand P, Kunnumakkara AB, Newman RA, Aggarwal BB. Bioavailability of curcumin: problems and promises. Mol Pharm. 2007;4(6):807-18.

6. Liang G, Yang S, Zhou H, Shao L, Huang K, Xiao J, et al. Synthesis, crystal structure and anti-inflammatory properties of curcumin analogues. Eur J Med Chem. 2009:44(2):915-9.

7. Zhao C, Yang J, Wang Y, Liang D, Yang X, Li X, et al. Synthesis of mono-carbonyl analogues of curcumin and their effects on inhibition of cytokine release in LPS-stimulated RAW 264.7 macrophages. Bioorg Med Chem. 2010;18(7):2388-93

8. Acharya A, Das I, Chandhok D, Saha T. Redox regulation in cancer: a double-edged sword with therapeutic potential. Oxid Med Cell Longev. 2010;3(1):23-34.

9. Cairns RA, Harris IS, Mak TW. Regulation of cancer cell metabolism. Nat Rev Cancer. 2011;11(2):85-95.

10. Circu ML, Aw TY. Reactive oxygen species, cellular redox systems, and apoptosis. Free Radic Biol Med. 2010;48(6):749-62.

11. Trachootham D, Alexandre J, Huang P. Targeting cancer cells by ROS-mediated mechanisms: a radical therapeutic approach? Nat Rev Drug Discov. 2009;8(7):579-91.

12. Zeng C, Zhong P, Zhao Y, Kanchana K, Zhang Y, Khan ZA, et al. Curcumin protects hearts from FFA-induced injury by activating Nrf2 and inactivating NF-KB both in vitro and in vivo. J Mol Cell Cardiol. 2015;79:1-12.

13. Zu K, Bihani T, Lin A, Park Y, Mori K, Ip C. Enhanced selenium effect on growth arrest by BiP/GRP78 knockdown in p53-null human prostate cancer cells. Oncogene. 2005;25(4):546-54.

14. Verfaillie T, Rubio N, Garg A, Bultynck G, Rizzuto R, Decuypere J, et al. PERK is required at the ER-mitochondrial contact sites to convey apoptosis after ROS-based ER stress. Cell Death Differ. 2012;19(11):1880-91.

15. Malumbres M, Barbacid M. Mammalian cyclin-dependent kinases. Trends Biochem Sci. 2005;30(11):630-41.

16. Zhang Z, Wang H, Li M, Agrawal S, Chen X, Zhang R. MDM2 is a negative regulator of p21WAF1/CIP1, independent of p53. J Biol Chem. 2004;279(16):16000-6.

17. Ando T, Kawabe T, Ohara H, Ducommun B, Itoh M, Okamoto T. Involvement of the interaction between p21 and proliferating cell nuclear antigen for the maintenance of G2/M arrest after DNA damage. Journal of Biological Chemistry. 2001;276(46):42971-7.

18. Schumacker PT. Reactive oxygen species in cancer cells: live by the sword, die by the sword. Cancer Cell. 2006;10(3):175-6.

19. Ketola K, Hilvo M, Hyötyläinen T, Vuoristo A, Ruskeepää A, Orešič M, et al. Salinomycin inhibits prostate cancer growth and migration via induction of oxidative stress. Br J Cancer. 2012;106(1):99-106.

20. Xiao D, Herman-Antosiewicz A, Antosiewicz J, Xiao H, Brisson M, Lazo JS, et al. Diallyl trisulfide-induced G2-M phase cell cycle arrest in human prostate cancer cells is caused by reactive oxygen species-dependent destruction and hyperphosphorylation of Cdc25C. Oncogene. 2005;24(41):6256-68.

21. Chen H-M, Chang F-R, Hsieh Y-C, Cheng Y-J, Hsieh K-C, Tsai L-M, et al. A novel synthetic protoapigenone analogue, WYC02-9, induces DNA damage and apoptosis in DU145 prostate cancer cells through generation of reactive oxygen species. Free Radical Biology and Medicine. 2011;50(9):1151-62.

22. Armstrong JL, Flockhart R, Veal GJ, Lovat PE, Redfern CP. Regulation of endoplasmic reticulum stress-induced cell death by ATF4 in neuroectodermal tumor cells. Journal of Biological Chemistry. 2010;285(9):6091-100.

23. Hill DS, Martin S, Armstrong JL, Flockhart R, Tonison JJ, Simpson DG, et al. Combining the endoplasmic reticulum stress-inducing agents bortezomib and fenretinide as a novel therapeutic strategy for metastatic melanoma. Clin Cancer Res. 2009;15(4):1192-8. 
24. Schwarze SR, Lin EW, Christian PA, Gayheart DT, Kyprianou N. Intracellular death platform steps-in: Targeting prostate tumors via endoplasmic reticulum (ER) apoptosis. Prostate. 2008;68(15):1615-23.

25. Verfaillie T, Garg AD, Agostinis P. Targeting ER stress induced apoptosis and inflammation in cancer. Cancer Lett. 2013;332(2):249-64.

26. Schröder M. Endoplasmic reticulum stress responses. Cell Mol Life Sci. 2008;65(6):862-94.

27. Szegezdi E, Logue SE, Gorman AM, Samali A. Mediators of endoplasmic reticulum stress-induced apoptosis. EMBO Rep. 2006;7(9):880-5.

28. Qu W, Xiao J, Zhang H, Chen Q, Wang Z, Shi H, et al. B19, a novel monocarbonyl analogue of curcumin, induces human ovarian cancer cell apoptosis via activation of endoplasmic reticulum stress and the autophagy signaling pathway. Int J Biol Sci. 2013;9(8):766.

29. Xiao J, Wang Y, Peng J, Guo L, Hu J, Cao M, et al. A synthetic compound, 1, 5-bis (2-methoxyphenyl) penta-1, 4-dien-3-one (B63), induces apoptosis and activates endoplasmic reticulum stress in non-small cell lung cancer cells. Int J Cancer. 2012;131(6):1455-65.

30. Liu Z, Sun Y, Ren L, Huang Y, Cai Y, Weng Q, et al. Evaluation of a curcumin analog as an anti-cancer agent inducing ER stress-mediated apoptosis in non-small cell lung cancer cells. BMC Cancer. 2013;13(1):494.

31. Geraghty P, Wallace A, D'Armiento JM. Induction of the unfolded protein response by cigarette smoke is primarily an activating transcription factor 4-C/EBP homologous protein mediated process. Int J Chron Obstruct Pulmon Dis. 2011;6:309.

32. Malhotra JD, Miao H, Zhang K, Wolfson A, Pennathur S, Pipe SW, et al. Antioxidants reduce endoplasmic reticulum stress and improve protein secretion. Proc Natl Acad Sci. 2008;105(47):18525-30.

33. Wang CC, Chiang Y-M, Sung S-C, Hsu Y-L, Chang J-K, Kuo P-L. Plumbagin induces cell cycle arrest and apoptosis through reactive oxygen species/C-Jun $\mathrm{N}$-terminal kinase pathways in human melanoma A375. S2 cells. Cancer Lett. 2008;259(1):82-98.

34. Boonstra J, Post JA. Molecular events associated with reactive oxygen species and cell cycle progression in mammalian cells. Gene. 2004;337:1-13.

\section{Submit your next manuscript to BioMed Central and take full advantage of:}

- Convenient online submission

- Thorough peer review

- No space constraints or color figure charges

- Immediate publication on acceptance

- Inclusion in PubMed, CAS, Scopus and Google Scholar

- Research which is freely available for redistribution 